\title{
Association between anthropometric measures and cardiovascular disease (CVD) risk factors in Hainan centenarians: investigation based on the Centenarian's health study
}

\author{
Qiao Zhu ${ }^{1 \dagger}$, Xiao-Bing Wang ${ }^{2+}$, Yao Yao ${ }^{1}$, Chao-Xue Ning ${ }^{1}$, Xiao-Ping Chen ${ }^{1}$, Fu-Xin Luan ${ }^{1 *}$ (i) and Ya-Li Zhao ${ }^{1 *}$
}

\begin{abstract}
Background: Centenarians refer to a special group who have outlived most of their fellows. Body shape and abdominal obesity have been identified as cardiovascular disease (CVD) risk factors. Our study aimed to evaluate the relationship between body mass index (BMI), waist circumference (WC), waist-to-hip ratio (WHR), and waist-to-height ratio (WHtR) and CVD risk factors among male and female centenarians in Hainan province.

Methods: Five hundred thirty-seven centenarians aged between 100 and 115 (Mage $=107$ years old) years participated in this study. Each participant received a standardized questionnaire and physical examination. We measured anthropometric variables (BMI, WC, WHR, WHtR, SBP and DBP) and serum lipid (TC, TG, HDL-C and LDL-C).

Results: $76.9 \%(n=413)$ of the study subjects were female. TC, TG, LDL-C and HDL-C were significantly higher in female group than that of male group. BMI, WC and WHtR were well-correlated with the CVD risk factors. The anthropometric measures were negatively related with HDL-C levels and positively related with the other CVD risk factors.
\end{abstract}

Conclusions: Hainan centenarians were short in stature and underweight. Moreover, female centenarians were often pear-shaped, while male centenarians were often apple-shaped. Further, BMI, WC and WHtR were well-correlated with the serum lipid, and TC, TG, LDL-C and HDL-C were significantly higher in females than males. Also, BMI, WC and WHtR were closely related to the incidence of dyslipidemia in females, including high TG, high LDL-C and low HDL-C.

Keywords: Centenarians, BMI, WC, WHR, WHtR, CVD risk factorts

\section{Background}

Centenarians represent a special group that deserves more attention. They have outlived most of their fellows. Data, anthropometric measures and biochemical indicators, from centenarians can be collected and used to study. So, our study aimed to elucidate the relationship between anthropometric measurements and cardiovascular disease (CVD) risk factors in Hainan centenarians.

\section{Methods}

Study population

Five hundred thirty-seven centenarians (413 female and 124 male) were enrolled in the study between June 2014 and October 2016 in Hainan. All participants provided written informed consent. The study protocol was approved by the ethical committee of the Hainan branch of PLA General Hospital (Sanya, China).

\section{Anthropometric measurements}

Anthropometric indices, Height $(\mathrm{H})$, Weight (W), waist circumference (WC) and hip circumference (HC), were measured with participants dressed in light clothing and

\footnotetext{
${ }^{+}$Equal contributors

${ }^{1}$ Hainan Branch of PLA General Hospital, Sanya 572000, China

Full list of author information is available at the end of the article
}

(c) The Author(s). 2018 Open Access This article is distributed under the terms of the Creative Commons Attribution 4.0 International License (http://creativecommons.org/licenses/by/4.0/), which permits unrestricted use, distribution, and 
Table 1 General Information of Study Subjects

\begin{tabular}{lllll}
\hline Variables & Total $(N=537)$ & Male $(N=124)$ & Female $(N=413)$ & $P . V a l u e$ \\
\hline Age & $102.6 \pm 2.8$ & $102.3 \pm 2.4$ & $102.8 \pm 2.9$ & 0.060 \\
Illiterate & 87.3 & 58.1 & 95.2 & $<0.001$ \\
Widowed & 88.8 & 62.9 & 93.5 & $<0.001$ \\
Han nationality & 83.6 & 80.6 & 84.5 & 0.309 \\
Smoker & 9.9 & 5.6 & 6.1 & 0.048 \\
Alcohol drinker & 13.6 & 25.8 & 9.9 & $<0.001$ \\
Tea drinker & 16.6 & 29.8 & 12.4 & $<0.001$ \\
No. of children & $4.2 \pm 2.2$ & $4.5 \pm 2.0$ & $4.1 \pm 2.3$ & 0.027 \\
\hline
\end{tabular}

$P$-value from Mann-Whitney $\mathrm{U}$ test for age and No. of children. Chisquare test for all other categorical variables. These tests were done to compare between males and females

Mean \pm standard deviation presented for continuous variables

barefoot. We measured the height and weight of the elderly with a scale (seca, Germany). Each parameter was measured twice. We computed the BMI, WHR and WHtR using the following standard formula: $\mathrm{BMI}=\mathrm{W} / \mathrm{H}^{2}, \mathrm{WHR}=\mathrm{WC} / \mathrm{HC}$, $\mathrm{WHtR}=\mathrm{WC} / \mathrm{H}$.

\section{Serum analysis}

Blood samples were analysed by the Hainan Branch of PLA General Hospital (Sanya, China). TC, TG, LDL-C and HDL-C were measured by electrochemiluminescence (Roche automatic biochemical analyser, cobas 6000, USA).

Table 2 Characteristics of Anthropometric Measurements and CVD Risk Factors

\begin{tabular}{|c|c|c|c|c|}
\hline Characteristics & Total $(n=537)$ & Male $(n=124)$ & Female $(n=413)$ & $P$-value \\
\hline \multicolumn{5}{|c|}{ Anthropometric measures } \\
\hline Height (m) & $146.00 \pm 9.37$ & $155.29 \pm 7.60$ & $143.21 \pm 7.94$ & $<0.001$ \\
\hline Weight (kg) & $38.45 \pm 8.72$ & $45.79 \pm 7.10$ & $36.24 \pm 7.92$ & $<0.001$ \\
\hline Waist (cm) & $75.49 \pm 9.17$ & $76.60 \pm 8.82$ & $75.15 \pm 9.26$ & 0.202 \\
\hline Hip (cm) & $83.96 \pm 7.05$ & $85.93 \pm 7.04$ & $83.37 \pm 6.96$ & $<0.001$ \\
\hline \multicolumn{5}{|c|}{ Anthropometric indices } \\
\hline BMl & $17.99 \pm 3.50$ & $18.99 \pm 2.66$ & $17.69 \pm 3.67$ & $<0.001$ \\
\hline WHR & $0.90 \pm 0.09$ & $0.89 \pm 0.07$ & $0.90 \pm 0.09$ & 0.241 \\
\hline $\mathrm{WHtR}$ & $0.52 \pm 0.07$ & $0.49 \pm 0.06$ & $0.53 \pm 0.07$ & $<0.001$ \\
\hline \multicolumn{5}{|c|}{ CVD Risk Factors measurements } \\
\hline $\mathrm{SBP}(\mathrm{mm} \mathrm{Hg})$ & $153.20 \pm 23.99$ & $148.93 \pm 21.35$ & $154.48 \pm 24.61$ & 0.024 \\
\hline $\mathrm{DBP}(\mathrm{mm} \mathrm{Hg})$ & $74.71 \pm 12.72$ & $73.50 \pm 11.78$ & $75.07 \pm 12.98$ & 0.230 \\
\hline $\mathrm{TC}(\mathrm{mg} / \mathrm{dl})$ & $135.05 \pm 28.36$ & $169.70 \pm 38.27$ & $185.89 \pm 37.49$ & $<0.001$ \\
\hline $\mathrm{TG}(\mathrm{mg} / \mathrm{dl})$ & $103.73 \pm 57.92$ & $94.11 \pm 41.12$ & $106.63 \pm 61.84$ & 0.012 \\
\hline $\mathrm{HDL}-\mathrm{C}(\mathrm{mg} / \mathrm{dl})$ & $55.36 \pm 14.72$ & $50.88 \pm 13.83$ & $56.71 \pm 14.73$ & $<0.001$ \\
\hline $\mathrm{LDL}-\mathrm{C}(\mathrm{mg} / \mathrm{dl})$ & $109.82 \pm 30.80$ & $101.87 \pm 29.62$ & $112.21 \pm 30.79$ & 0.001 \\
\hline \multicolumn{5}{|c|}{ Prevalence of CVD Risk Factors, n (\%) } \\
\hline Hypertension & $164.58 \pm 17.48$ & $160.44 \pm 14.62$ & $181.74 \pm 12.71$ & 0.006 \\
\hline High TC & $265.78 \pm 22.17$ & $263.56 \pm 20.50$ & $266.27 \pm 22.80$ & 0.774 \\
\hline High TG & $280.73 \pm 147.83$ & $220.03 \pm 17.92$ & $294.22 \pm 160.87$ & 0.035 \\
\hline Low HDL-C & $34.92 \pm 4.20$ & $34.46 \pm 4.31$ & $35.26 \pm 4.14$ & 0.432 \\
\hline High LDL-C & $179.71 \pm 20.20$ & $175.43 \pm 13.98$ & $180.52 \pm 21.25$ & 0.579 \\
\hline
\end{tabular}

$P$-value from two independent samples t-test for WHR, Diastolic Blood Pressure, Hypertension, High Total Cholesterol, High Triglycerides, Low HDL-C and High LDL-C. Mann-Whitney U test for all other continuous variables. These tests were done to compare between males and females

Mean \pm standard deviation presented continuous variables

CVD RF Cardiovascular disease risk factors 
Table 3 Spearman Correlation Coefficient between Anthropometric Indices and Cardiovascular Disease Risk Factors

\begin{tabular}{cllll}
\hline Variables & BMl & WC & WHR & WHtR \\
\hline Overall $(n=537)$ & & & & \\
SBP & $0.115^{* *}$ & $0.124^{* *}$ & 0.070 & $0.114^{* *}$ \\
DBP & 0.082 & $0.139^{* *}$ & 0.035 & $0.095^{*}$ \\
TC & $0.097^{*}$ & $0.108^{*}$ & 0.058 & $0.138^{* *}$ \\
TG & $0.219^{* *}$ & $0.178^{* *}$ & 0.032 & $0.171^{* *}$ \\
HDL-C & $-0.166^{* *}$ & $-0.123^{* *}$ & -0.023 & -0.044 \\
LDL-C & $0.160^{* *}$ & $0.160^{* *}$ & 0.075 & $0.161^{* *}$ \\
Male (124) & & & & \\
SBP & 0.055 & 0.170 & 0.073 & 0.164 \\
DBP & -0.023 & $0.228^{*}$ & 0.063 & 0.147 \\
TC & 0.122 & 0.040 & -0.030 & 0.118 \\
TG & $0.268^{* *}$ & $0.179^{*}$ & 0.033 & $0.195^{*}$ \\
HDL-C & -0.156 & -0.075 & -0.094 & -0.015 \\
LDL-C & $0.188^{*}$ & 0.101 & -0.017 & 0.137 \\
Female (413) & & & & \\
SBP & $0.162^{* *}$ & $0.120^{*}$ & 0.068 & 0.079 \\
DBP & $0.122^{*}$ & $0.119^{*}$ & 0.029 & 0.074 \\
TC & $0.131^{* *}$ & $0.151^{* *}$ & 0.082 & $0.108^{*}$ \\
TG & $0.226^{* *}$ & $0.183^{* *}$ & 0.022 & $0.142^{* *}$ \\
HDL-C & $-0.132^{* *}$ & $-0.126^{*}$ & -0.014 & $-0.104^{*}$ \\
LDL-C & $0.188^{* *}$ & $0.194^{* *}$ & $0.097^{*}$ & $0.141^{* *}$ \\
\hline The associtins & & & & \\
\end{tabular}

The associations between anthropometric measures and CVD risk factors were examined using Spearman's correlation coefficients

** All are significant at the level of $<0.01$ (2-tailed)

${ }^{*}$ All are significant at the level of $<0.05$ (2-tailed)

\section{Blood pressure measurement}

Two blood pressure recordings were measured by electronic sphygmomanometers (Omron HEM-7200, Japan). If the difference between the first and second measurement was more than $5 \mathrm{mmHg}$, the repeated measurements were performed.

\section{Definition of CVD risk factors}

Hypertension was defined as having one or more of the following: (1) a systolic BP $\geq 140 \mathrm{mmHg}$, (2) a diastolic $\mathrm{BP} \geq 90 \mathrm{mmHg}$, (3) physician-diagnosed hypertension.
Dyslipidemia was defined according to the Chinese guidelines on the prevention and treatment of dyslipidemia in adults (2007): TC $\geq 240 \mathrm{mg} / \mathrm{dl}$ as high; LDL-C $\geq$ $160 \mathrm{mg} / \mathrm{dl}$ as high; HDL-C $<40 \mathrm{mg} / \mathrm{dl}$ as low; and TG $\geq$ $200 \mathrm{mg} / \mathrm{dl}$ as high.

\section{Statistical analysis}

We used EpiData 3.0 software to double-enter the data, and SPSS 19.0 software (SPSS Inc., Chicago, IL, USA) was used for statistical analysis. Continuous variables were tested for normality using the Kolmogorov-Smirnov test, and chisquare test for categorical variables. Comparisons between males and females were performed using two independent samples t-test or the Mann-Whitney $U$ test for continuous variables. The associations between anthropometric measures and CVD risk factors were firstly examined using Spearman's correlation coefficients. Receiver Operating Characteristic (ROC) analyses were then used to calculate the area under the ROC curves (AUC). Multiple logistic regressions were used in female centenarians at last. $p<0.05$ indicated statistical significance.

\section{Results}

Characteristics of study subjects

We collected 537 centenarians' personal information (Table 1). There were a statistically significant difference in illiteracy, widowed, alcohol and tea drinking between male and female centenarians. Anthropometric measurements and CVD risk factors are shown in Table 2. Differences on BMI and WHtR were significant. With regards to serum lipids, TC, TG, LDL-C and HDL-C were significantly higher in female group than that of male group. Moreover, the difference was statistically significant in SBP between men and women. Correspondingly, the prevalence of hypertension and high TG were significantly higher in females than males.

\section{Correlations between anthropometric measures and CVD risk factor variables}

The gender-stratified Spearman's coefficients for the correlations between the various anthropometric measures and CVD risk factors are shown in Table 3. BMI, WC and WHtR were well-correlated with the various CVD risk factors. The anthropometric measures were negatively

Table 4 Adjusted Area Under Receiver Operating Characteristic (ROC) Curve for the Various Anthropometric Indices and Cardiovascular Disease Risk Factors

\begin{tabular}{|c|c|c|c|c|}
\hline Variables & BMI & WC & WHR & $\mathrm{WHtR}$ \\
\hline Hypertension & $0.546(0.492-0.600)$ & $0.551(0.496-0.605)$ & $0.536(0.481-0.590)$ & $0.531(0.478-0.585)$ \\
\hline High TC & $0.530(0.448-0.611)$ & $0.548(0.457-0.639)$ & $0.529(0.433-0.626)$ & $0.527(0.434-0.620)$ \\
\hline High TG & $0.698(0.591-0.805)$ & $0.638(0.519-0.757)$ & $0.475(0.365-0.586)$ & $0.631(0.520-0.742)$ \\
\hline Low HDL-C & $0.661(0.595-0.728)$ & $0.578(0.502-0.654)$ & $0.536(0.463-0.609)$ & $0.513(0.438-0.588)$ \\
\hline High LDL-C & $0.577(0.499-0.654)$ & $0.574(0.482-0.666)$ & $0.516(0.420-0.611)$ & $0.554(0.459-0.649)$ \\
\hline
\end{tabular}

Anthropometric measure with the highest AUC value in bold 


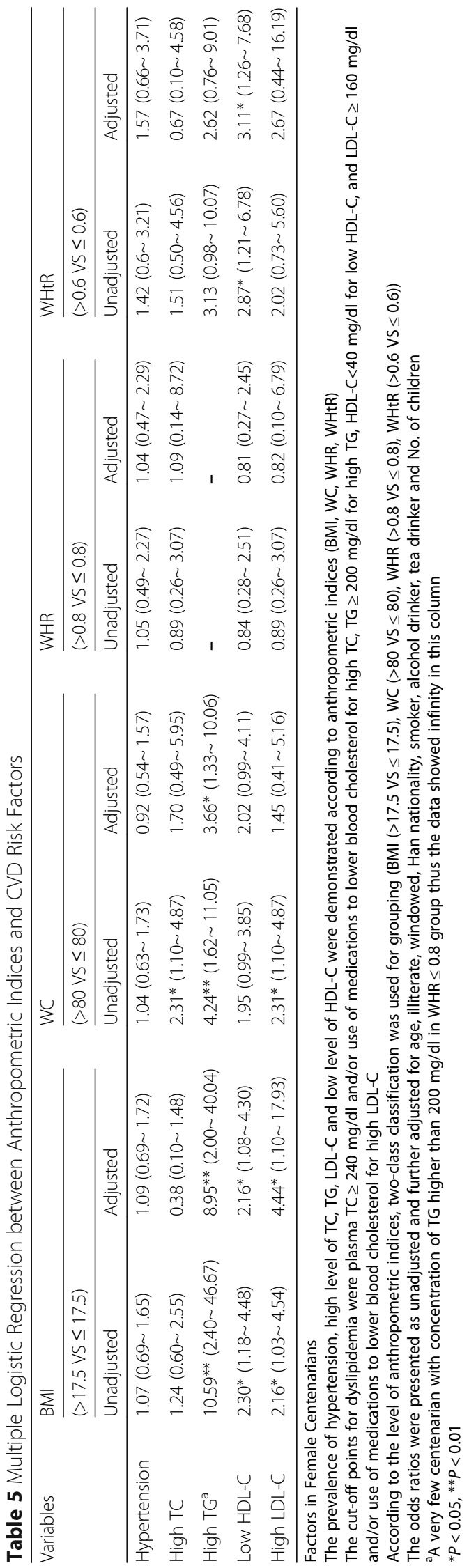


related with HDL-C levels and positively related with the other CVD risk factors. However, the relationship is not fairly distributed between women and men.

\section{Association of various anthropometric measures and CVD risk factors using ROC curve analyses}

The area under the ROC curves (AUCs) for the association between CVD risk factors and anthropometric measures are shown in Table 4. BMI and WC were associated with the highest AUCs for two of the five CVD risk factors (high TG and low HDL-C), and WHtR was associated with the highest AUC for high TG. However, the differences in the AUCs for the various anthropometric measures were often small, demonstrating overlapping 95\% confidence intervals (CIs).

\section{Association of various anthropometric indices and CVD risk factors in female centenarians}

Multiple logistic regressions were used to evaluate the association between anthropometric indices and CVD risk factors in female centenarians, as shown in Table 5. The incidence of high TG, low HDL-C and high LDL-C were closely related to BMI, 10.59 times, 2.30 times and 2.16 times, respectively. Besides, high TG is also associated with WC (4.24 times unadjusted and 3.66 times adjusted). Furthermore, low HDL is associated with WHtR (2.87 times unadjusted and 3.11 times adjusted). However, it should be noted that there were a very few centenarians with high TG with WHR $\leq 0.8$. So, the data in this column showed infinity.

\section{Discussion}

Cardiovascular diseases (CVD) have become serious causes of death among old people. Dyslipidemia is one of the most important independent risk factors for CVD $[1,2]$. Increasing the awareness and management of patients with dyslipidemia has a positive impact on CVD prevention.

This study was conducted in Hainan to comprehensive investigate the physical condition of the centenarians as well as the associated CVD risk factors. Our results indicated that the difference was statistically significant in TC, TG, LDL-C and HDL-C between males and females. It was also revealed that BMI, WC and WHtR were negatively related with $\mathrm{HDL}-\mathrm{C}$ levels and positively related with the other CVD risk factors. Furthermore, our results suggested that the presence of dyslipidemia was significantly associated with body size.

Study has shown that the longevity of people has its own characteristics, such as short stature, light weight and more. Consistent with prior studies, our results showed that Hainan centenarians were short in stature (female 143.21 $\pm \pm 7.94 \mathrm{~cm}$, male $155.29 \pm 7.60 \mathrm{~cm}$ ), underweight (female $36.24 \pm 7.92 \mathrm{~kg}$, male $45.79 \pm 7.10 \mathrm{~kg}$ ), and
BMI $(17.99 \pm 3.50)$ was far lower than the cut-off values for Asian populations.

Generally, abdominal fat was positively correlated with cardiovascular risk factors. Much research has been proved that the people in the Asian-pacific regions are easy to gain the abdominal obesity. In the study, we observed that female centenarians were often pear-shaped (WHR $\geq 0.8$ for women), while males were often appleshaped (WHR $<0.9$ for men). Our results were in line with previous studies [3, 4]. It has been proposed that circulating gonadal steroids determine these sex-specific differences in adipose tissue distribution, which can be observed even after menopause [5]. Moreover, women's adipose tissue is more favourable to accumulate in the peripheral and gluteofemoral depots to prevent the development of atherosclerosis [6-8]. In a word, the fat distribution pattern of women contributes to more healthier metabolic status, and may even take part in the determination of the overall life expectancy.

On the other hand, studies reported that age was one of major risk factors of dyslipidemia, which may be related to hereditary characteristics and degenerative processes as well as adipose tissue distribution and progressive development of insulin resistance $[9,10]$. In our study, BMI, WC and WHtR were well-correlated with the serum lipid, and TC, TG, LDL-C and HDL-C were significantly higher in females than males. This may be associated with changes in postmenopausal hormone levels [11]. oestrogen levels are lower, glucose and lipid metabolism may differ significantly $[12,13]$. In addition, we observed that BMI and WC were exhibited the relationship with high TG and low HDL-C. This was consistent with some previous studies $[9,11,14]$. Interestingly, the results using multiple logistic regressions indicated that BMI, WC and WHtR were closely related to the incidence of dyslipidemia in femals centenarians, including high TG, high LDL-C and low HDL-C. This suggests that body measurement indices are important non-invasive indicators in evaluating health, which is used to assess lipid metabolism abnormalities. Unfortunately, due to the insufficient number of male centenarians, we failed to complete the multiple logistic regression analysis. We still need to continue our investigation and preserve these precious data to further guide our research.

\section{Conclusion}

Hainan centenarians were short in stature and underweight. Moreover, female centenarians were often pear-shaped, while male centenarians were often apple-shaped. Further, BMI, WC and WHtR were well-correlated with the serum lipid, and TC, TG, LDL-C and HDL-C were significantly higher in females than males. Also, BMI, WC and WHtR were closely related to the incidence of dyslipidemia in females, including high TG, high LDL-C and low HDL-C. 


\section{Abbreviations}

BMI: Body mass index; CVD: Cardiovascular disease; DBP: Diastolic blood pressure; HC: Hip circumference; HDL-C: High-density lipoprotein cholesterol; LDL-C: Low-density lipoprotein cholesterol; SBP: Systolic blood pressure; TC: Total cholesterol; TG: Triglyceride; WC: Waist circumference; WHR: Waist-hip ratio; WHtR: Waist-to-height ratio

\section{Funding}

The study was funded by Key Research and Development Project of Hainan (NO. ZDYF2016135, ZDYF2017095) and Natural Science Foudation of Hainan (NO. 817360).

\section{Availability of data and materials}

The datasets used and analysed during the current study available from the corresponding author on reasonable request.

\section{Authors' contributions}

ZQ and WXB performed the statistical analysis and drafted the manuscript. YY participated in the design of the study and performed the investigation. NCX performed the statistical analysis and made substantial contributions to acquisition and analysis of data. CXP performed data entry and sample management. LFX and ZYL conceived the study, participated in its design and coordination, and helped draft the manuscript. All authors read and approved the final manuscript.

\section{Ethics approval and consent to participate}

The experimental protocol was established, according to the ethical guidelines of the Helsinki Declaration and was approved by the Human Ethics Committee of Hainan Branch of PLA General Hospital, Sanya, China. Written informed consent was obtained from individual participants.

\section{Competing interests}

The authors declare that they have no competing interests.

\section{Publisher's Note}

Springer Nature remains neutral with regard to jurisdictional claims in published maps and institutional affiliations.

\section{Author details}

'Hainan Branch of PLA General Hospital, Sanya 572000, China. ${ }^{2}$ Department of Military Education and Training, Naval Aeronautical and Astronautical University, Yantai 264001, China.

Received: 19 September 2017 Accepted: 20 April 2018

Published online: 02 May 2018

\section{References}

1. Li JH, Wang LM, Mi SQ, Zhang M, Li YC, Jiang Y, Xu Y, Dai M, Wang LH. Awareness rate, treatment rate and control rate of dyslipidemia in Chinese adults, 2010. Zhonghua Yu Fang Yi Xue Za Zhi. 2012;46(8):687-91.

2. Luo JY, YT Ma ZXY, Yang YN, Xie X, Ma X, Liu F, Li XM, Chen BD. Prevalence, awareness, treatment and control of dyslipidemia among adults in northwestern China: the cardiovascular risk survey. Lipids Health Dis. 2014 13:4. https://doi.org/10.1186/1476-511x-13-4.

3. Zhao X, Zhu X, Zhang H, Zhao W, Li J, Shu Y, Li S, Yang M, Cai L, Zhou J, L $Y$. Prevalence of diabetes and predictions of its risks using anthropometric measures in southwest rural areas of China. BMC Public Health. 2012;12:821. https://doi.org/10.1186/1471-2458-12-821.

4. Karastergiou K, Smith SR, Greenberg AS, Fried SK. Sex differences in human adipose tissues - the biology of pear shape. Biol Sex Differ. 2012;3(1):13. https://doi.org/10.1186/2042-6410-3-13.

5. Fuente-Martin E, Argente-Arizon P, Ros P, Argente J, Chowen JA. Sex differences in adipose tissue: it is not only a question of quantity and distribution. Adipocyte. 2013;2(3):128-34. https://doi.org/10.4161/adip.24075.

6. Tanko LB, Bagger YZ, Alexandersen P, Larsen PJ, Christiansen C. Central and peripheral fat mass have contrasting effect on the progression of aortic calcification in postmenopausal women. Eur Heart J. 2003:24(16):1531-7.

7. Lissner L, Bjorkelund C, Heitmann BL, Seidell JC, Bengtsson C. Larger hip circumference independently predicts health and longevity in a Swedish female cohort. Obes Res. 2001;9(10):644-6. https://doi.org/10.1038/oby.2001.85
8. Tanko LB, Bagger YZ, Alexandersen P, Larsen PJ, Christiansen C. Peripheral adiposity exhibits an independent dominant antiatherogenic effect in elderly women. Circulation. 2003;107(12):1626-31. https://doi.org/10.1161/01.cir 0000057974.74060 .68 .

9. Bayram F, Kocer D, Gundogan K, Kaya A, Demir O, Coskun R, Sabuncu T, Karaman A, Cesur M, Rizzo M, Toth PP, Gedik V. Prevalence of dyslipidemia and associated risk factors in Turkish adults. J Clin Lipidol. 2014;8(2):206-16. https://doi.org/10.1016/j.jacl.2013.12.011.

10. Ni WQ, Liu XL, Zhuo ZP, Yuan XL, Song JP, Chi HS, Xu J. Serum lipids and associated factors of dyslipidemia in the adult population in Shenzhen. Lipids Health Dis. 2015;14:71. https://doi.org/10.1186/s12944-015-0073-7.

11. Sharma U, Kishore J, Garg A, Anand T, Chakraborty M, Lali P. Dyslipidemia and associated risk factors in a resettlement colony of Delhi. J Clin Lipidol. 2013;7(6):653-60. https://doi.org/10.1016/j.jacl.2013.06.003.

12. Hong SC, Yoo SW, Cho GJ, Kim T, Hur JY, Park YK, Lee KW, Kim SH. Correlation between estrogens and serum adipocytokines in premenopausal and postmenopausal women. Menopause. 2007:14(5):83540. https://doi.org/10.1097/GME.0b013e31802cddca.

13. Mattsson C, Olsson T. Estrogens and glucocorticoid hormones in adipose tissue metabolism. Curr Med Chem. 2007:14(27):2918-24.

14. Shen Z, Munker S, C Wang LX, Ye H, H Chen GX, Zhang H, Chen L, Yu C, Li Y Association between alcohol intake, overweight, and serum lipid levels and the risk analysis associated with the development of dyslipidemia. J Clin Lipidol. 2014;8(3):273-8. https://doi.org/10.1016/j.jacl.2014.02.003.

\section{Ready to submit your research? Choose BMC and benefit from:}

- fast, convenient online submission

- thorough peer review by experienced researchers in your field

- rapid publication on acceptance

- support for research data, including large and complex data types

- gold Open Access which fosters wider collaboration and increased citations

- maximum visibility for your research: over $100 \mathrm{M}$ website views per year

At BMC, research is always in progress.

Learn more biomedcentral.com/submissions 